\title{
Ginsenoside Rb1 Protects the Brain from Damage Induced by Epileptic Seizure via Nrf2/ARE Signaling
}

\author{
Yunbo Shi ${ }^{a} \quad$ Wang Miao ${ }^{a} \quad J u n f a n g$ Teng $^{a} \quad$ Lingli Zhang $^{b}$ \\ aDepartment of Neurology, The First Affiliated Hospital of Zhengzhou University, Zhengzhou, \\ bDepartment of Gastroenterology, The First Affiliated Hospital of Zhengzhou University, Zhengzhou, \\ China
}

\section{Key Words}

Ginsenoside Rb1 • Epilepsy • Nrf2-ARE • Heme oxygenase-1 (HO-1) • Pentylenetetrazole (PTZ)

\begin{abstract}
Background/Aims: Ginsenoside $\mathrm{Rb} 1$ (Rb1) has been reported to have varieties of neuroprotective effects. This study aimed to evaluate the effects of Rb1 on pentylenetetrazol (PTZ)-induced rat brain injury and $\mathrm{Mg}^{2+}$ free-induced neuron injury and analyzed the detailed molecular mechanisms in vivo and in vitro. Methods: Seizure duration and latency were measured in epilepsy kindled rat. The cognitive impairment was assessed by Morris water maze (MWM) test. Oxidative stress parameters, malondialdehyde (MDA) and glutathione (GSH) were measured by the 2-thiobarbituric acid methods and the DTNB-GSSG reductase recycling methods. Neuronal damage was assessed by hematoxylin and eosin (H\&E) and Nissl staining. Neuronal apoptosis was measured by Annexin V-FITC and propidium iodide (PI) staining. Immunohistochemistry and immunofluorescence staining were performed to evaluate Nrf2 and HO-1 expressions. Expression of Nrf2, $\mathrm{HO}-1, \mathrm{BCl}-2$, iNOS and LC3 were evaluated by western blot. Results: The PTZ-injured rats presented longer seizure duration and shorter seizure latency. Rb1 ameliorated these effects, as well as the cognitive deficits caused by PTZ exposure. Besides, Rb1 dose-dependently increased GSH levels, decreased MDA levels and alleviated neuronal damage in PTZ-treated rats. In vitro, Rb1 increased cell viability and decreased neuronal apoptosis in a dose-dependent manner under $\mathrm{Mg}^{2+}$ free condition. Moreover, in vivo and in vitro, $\mathrm{Rb} 1$ enhanced both the Nrf2 and $\mathrm{HO}-1$ expressions. Furthermore, upregulation of the expression of $\mathrm{Bcl}-2$ and downregulation of the expression of iNOS and LC3 were observed. However, knockdown of Nrf2 adversely affected the protective effects of Rb1 in epileptic hippocampal neurons. Conclusion: Rb1 conferred neuroprotective effects against PTZ-induced brain damage and $\mathrm{Mg}^{2+}$ free-induced neuron injury by activating Nrf2/ARE signaling.




\section{Introduction}

Epilepsy is a major complex neurological disorder, which is characterized by recurrent seizures due to an imbalance between cerebral excitability and inhibition [1]. Epilepsy affects about $1 \%$ of the population worldwide and has become a serious global public health issue [2]. The underlying pathophysiological mechanisms of epilepsy are still incompletely understood. The environmental, genetic and epigenetic factors are interwoven in individual patients with different types of epilepsy [3]. Besides, oxidative stress and impaired autophagy contribute to epileptogenesis $[4,5]$. However, there is no effective treatment to prevent the genesis or progression of seizures [6]. Therefore, finding a more effective drug for epilepsy would provide novel targets for the therapeutic strategies [7].

Ginsenoside $\mathrm{Rb} 1$ ( $\mathrm{Rb} 1$ ), a principle bioactive ingredients in ginseng, is isolated from the roots of Panax ginseng C.A. Meyer [8]. Rb1 has received much attention due to its biological functions, especially the various neuroprotective effects [9]. Increasing evidence demonstrates that $\mathrm{Rb} 1$ can protect against neuronal injury with the properties of antiinflammation, anti-apoptosis and induction of neurogenesis [10-12]. Besides, recent studies have also indicated that $\mathrm{Rb} 1$ can increase cell genesis in hippocampal subregions to improve the spatial learning and memory of rats [13]. However, the neuroprotective properties of $\mathrm{Rb} 1$ in epilepsy are still elusive.

The NF-E2 related factor 2/antioxidant response element (Nrf2/ARE) signaling pathway is an endogenous cytoprotective system $[14,15]$. Once activated, the transcription factor Nrf2 translocates to the nucleus of the cell, where it forms heterodimers with other transcription factors such as c-Jun, and then binds to the ARE [16]. Nrf2-ARE binding regulates more than 200 genes, which are involved in the cellular anti-inflammatory and antioxidant defense, such as NAD(P)H quinone oxidoreductase 1 (NQO1) and heme oxygenase-1 (HO-1) [1719]. In addition, activation of the Nrf2/ARE pathway plays important roles in animal and cell culture models of neurodegenerative diseases, including Parkinson disease, Alzheimer disease and epilepsy [20-22].

In the present study, we developed a rat model of epilepsy induced by PTZ, in vivo. Meanwhile, an in vitro model of hippocampal neurons cultured in $\mathrm{Mg}^{2+}$ free medium was established. The primary goal was to evaluate the effects of Rb1 on PTZ-induced rat brain injury and $\mathrm{Mg}^{2+}$ free-induced neuron injury and analyze the detailed molecular mechanisms in vivo and in vitro.

\section{Materials and Methods}

\section{Animals}

Healthy male Sprague-Dawley (SD) rats (220 $240 \mathrm{~g})$ were provided by the Animal Experimental center of Zhengzhou University. The protocols were approved by the Institutional Animal Ethics Committee of The First Affiliated Hospital of Zhengzhou University. Animals were handled according to the guidelines of the Care and Use of Laboratory Animals by the National Institute of Health, China.

\section{Model establishment and grouping}

Rats were assigned randomly into six experimental groups ( $n=6$ in each group) as follows: (1) Normal group: rats were intraperitoneally injected with $1 \mathrm{ml}$ of normal saline, every $48 \mathrm{~h}$; (2) PTZ group: the rats were intraperitoneally injected with a single dose of $60 \mathrm{mg} / \mathrm{kg}$ PTZ (Sigma, St. Louis, USA) dissolved in $1 \mathrm{ml}$ of normal saline, every $48 \mathrm{~h}$ till to $26 \mathrm{~d}$; (3) PTZ+0.9\% NaCl group: a group treated with $10 \mathrm{~mL} / \mathrm{kg}$ saline $10 \mathrm{~min}$ before PTZ treatment; (4) PTZ+low dose: rats were intraperitoneally injected with $15 \mathrm{mg} /$ $\mathrm{kg} \mathrm{Rb1} \mathrm{(1.5} \mathrm{mg/mL,} \mathrm{dissolved} \mathrm{in} \mathrm{saline,} 10 \mathrm{~mL} / \mathrm{kg}$; Fleton Natural Products, Chengdu, China) $10 \mathrm{~min}$ before PTZ treatment; (5) PTZ+mid dose: rats were intraperitoneally injected with $30 \mathrm{mg} / \mathrm{kg} \mathrm{Rb1} \mathrm{(3} \mathrm{mg/mL,}$ dissolved in saline, $10 \mathrm{~mL} / \mathrm{kg}$ ) $10 \mathrm{~min}$ before PTZ treatment; (6) PTZ+high dose: rats were intraperitoneally injected with $60 \mathrm{mg} / \mathrm{kg} \mathrm{Rb} 1$ (6 mg/mL, dissolved in saline, $10 \mathrm{~mL} / \mathrm{kg}$ ) $10 \mathrm{~min}$ before PTZ treatment. Rb1 was administered intraperitoneally (i.p.) daily for $26 \mathrm{~d}$. After treatment for $26 \mathrm{~d}$, the rats were monitored. 


\section{Cellular Physiology Cell Physiol Biochem 2018;45:212-225

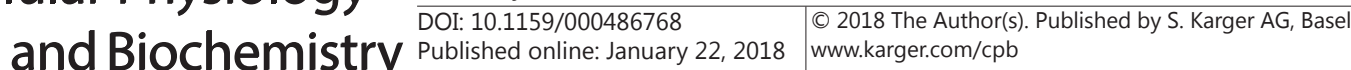

Shi et al.: Ginsenoside Rb1, a Novel Anti-Epileptic Drug

Behavioural seizure responses were scored according to Racine grading standard [23]: grade 0, no reaction; grade I, facial twitches (lips, nose and eyes); grade II, head nodding; grade III, unilateral forelimb clonus; grade IV, rearing with bilateral forelimb clonus; grade V, imbalance and falling on side or back. The latencies were recorded as the duration from the injection of drug to behavioural seizure (grade V).

\section{Morris water maze test}

After $26 \mathrm{~d}$, morris water maze test was used to evaluate the cognitive function of rats. SuperMaze Morris (XR-XM101; Xinruan, Shanghai, China) consisted of a circular water pool diameter $120 \mathrm{~cm}$, height 50 $\mathrm{cm}$ and filled with water $\left(22 \pm 1^{\circ} \mathrm{C}\right)$. Besides, a platform $(10 \mathrm{~cm}$ in diameter $)$ was located in a labelled target quadrant $2 \mathrm{~cm}$ below surface. A digital camera connected to the computer was above the pool.

Then the rats were released into the water from an assigned release point. For each test, rats were allowed to swim until they found and landed on the platform. The time that the rats used to find the platform (i.e. the escape latency) was recorded. If the rat failed to find the platform within $90 \mathrm{~s}$, they would be picked up and placed on the platform for $10 \mathrm{~s}$, the escaping latency was recorded as $90 \mathrm{~s}$. This test was repeated 4 times. Swimming distance and speed were recorded. Data were analyzed by MWM software (Watermaze 3.31, Actimetrics, IL, USA).

\section{Tissue processing}

After the morris water maze test, rats were sacrificed by deeply anesthetized with isoflurane $(0.75 \%)$. Brains were then removed, embedded in paraffin and then cut into $5 \mu \mathrm{m}$-thick coronal sections for H\&E staining, Nissl staining, immunohistochemical analysis and immunofluorescence analysis. Additional tissues were snap frozen in liquid nitrogen and stored at $-80^{\circ} \mathrm{C}$ until further use.

\section{H\&E staining}

H\&E Staining was performed according to previous studies [24]. In brief, the CA1 region of the hippocampus was stained with hematoxylin for $15 \mathrm{~min}$ and hydrochloric acid alcohol solution for $35 \mathrm{~s}$. Then the sections were stained with eosin for $10 \mathrm{~min}$ and $90 \%$ ethanol for $40 \mathrm{~s}$. After that, the section was observed and photographed under the microscope.

\section{Nissl staining}

Nissl Staining was performed according to previous studies [25]. The CA1 region of the hippocampus was deparaffinized and rinsed with distilled water three times and then stained with $0.25 \%$ toluidine blue at $50^{\circ} \mathrm{C}$ for $3 \mathrm{~h}$. Then the sections were bleached using 95\% ethanol. After $100 \%$ ethanol dehydration and xylene transparency, sections were observed under a microscope (BX53, Olympus).

\section{Immunohistochemical analysis}

The immunohistochemical staining was performed according to the kit instructions (Boster Biological Company, China). Briefly, tissue sections underwent dehydration through the ethanol gradient. Then endogenous peroxidase activity was blocked by $3 \mathrm{H}_{2} \mathrm{O}_{2}$ for $40 \mathrm{~min}$ at room temperature. After incubation in normal rabbit serum for $40 \mathrm{~min}$, they were incubated with the appropriate primary antibody (Nrf2 and HO-1; Wuhan Boster Biological Technology, Wuhan, China) in a dilution of 1: 200 overnight at $4^{\circ} \mathrm{C}$. Subsequently, they were treated with the HRP conjugated IgG (1:500) for $60 \mathrm{~min}$ at room temperature. The immune reaction was visualized by incubated with DAB for $5 \mathrm{~min}$. Sections were then examined using light microscopy.

\section{Immunofluorescence analysis}

Immunofluorescence assay was performed according to previous studies [26]. Briefly, tissue sections underwent dehydration through the ethanol gradient. Then the sections were blocked by $5 \%$ normal rabbit serum at room temperature for $30 \mathrm{~min}$ and $0.3 \%$ Triton X-100 for $15 \mathrm{~min}$. The sections were followed by incubation with appropriate primary antibody (Nrf2 and HO-1; Wuhan Boster Biological Technology, Wuhan, China) in a dilution of 1: 100 overnight at $4^{\circ} \mathrm{C}$. After washing three times with PBS, the sections were incubated with secondary antibodies for $30 \mathrm{~min}$ at $37^{\circ} \mathrm{C}$. The secondary antibodies were Alexa fluorconjugated anti-mouse IgG2b (Molecular Probes, 1:200) and fluorescein isothiocyanate (FITC)-conjugated anti-rabbit IgG (DAKO Corp, 1:100) for the detection of the expression of Nrf2 and HO-1. Subsequently, 


\section{Cellular Physiology Cell Physiol Biochem 2018;45:212-225

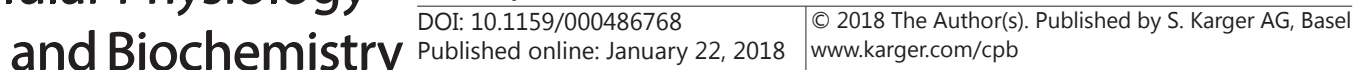

Shi et al.: Ginsenoside Rb1, a Novel Anti-Epileptic Drug

the sections were counter-stained with 4, 6-diamidino-2-phenylindole (DAPI). Immunofluorescent images were visualized using confocal microscope (FV1000, Olympus).

\section{MDA and GSH assay}

Brain tissues were homogenized and centrifuged for supernatant collection. MDA and GSH assays were performed according to the manufacturer's protocols (Nanjing Jiancheng, China). MDA levels were measured using 2-thiobarbituric acid methods. Besides, GSH levels were analyzed using the DTNB-GSSG reductase recycling methods. Subsequently, colorimetric methods were used for the assays and the results were calculated using the absorbance values and standard equations.

\section{Primary neuronal cell cultures}

The culture of hippocampal neurones were performed using the methods described previously [27]. In brief, hippocampal neuronal cells were obtained from rats 1-3 days old. The cells were dissociated in Hanks' balanced salt solution supplemented with $0.125 \%$ trypsin (Sigma-Aldrich, USA) for $10 \mathrm{~min}$ at $37^{\circ} \mathrm{C}$. The cells were then cultured in Dulbecco's modified Eagle's medium (DMEM, Invitrogen Life Technologies, USA) containing $10 \%$ fetal bovine serum and penicillin-streptomycin (100 U/ml, Gibco-BRL). After cultivation for 2 weeks, the cells were used in the following experiments.

\section{Establishment of the epileptic cell model}

The in vitro epileptic cell model was established according to a conventional method [28]. In brief, hippocampal neurons were cultured in nutrient medium for $9 \mathrm{~d}$. Then the nutrient medium was changed into magnesium ion $\left(\mathrm{Mg}^{2+}\right)$ free extracellular medium for $3 \mathrm{~h}$. The solution contained $145 \mathrm{mM} \mathrm{NaCl}, 2.5 \mathrm{mM}$ $\mathrm{KCl}, 2 \mathrm{mM} \mathrm{CaCl}, 10 \mathrm{mM}$ HEPES, $10 \mathrm{mM}$ glucose and $0.002 \mathrm{mM}$ glycine (pH 7.2). Besides, the osmolarity was adjusted to $290 \pm 10 \mathrm{mOsm}$ using sucrose. This can induce permanently manifested recurrent, spontaneous seizure discharges characteristic of the same electrographic properties seen in human epilepsy [29]. The cells cultured in normal extracellular culture medium $\left(145 \mathrm{mM} \mathrm{NaCl}, 2.5 \mathrm{mM} \mathrm{KCl}, 2 \mathrm{mM} \mathrm{CaCl}{ }_{2}, 10 \mathrm{mM}\right.$ HEPES, $10 \mathrm{mM}$ glucose, $0.002 \mathrm{mM}$ glycine ( $\mathrm{pH} 7.2$ ), $1 \mathrm{mM} \mathrm{MgCl}_{2}$ and $290 \pm 10 \mathrm{mOsm}$ ) for $3 \mathrm{~h}$ were used as control.

\section{Cell grouping}

The cells were divided into groups: (1) Normal+low does group: The cells were pre-treated with $5 \mu \mathrm{M}$ $\mathrm{Rb} 1$ for $24 \mathrm{~h}$, and then the cells were cultured in normal extracellular culture medium for $3 \mathrm{~h}$. (2) Normal+mid does group: The cells were pre-treated with $10 \mu \mathrm{M} \mathrm{Rb} 1$ for $24 \mathrm{~h}$, and then the cells were cultured in normal extracellular culture medium for $3 \mathrm{~h}$. (3) Normal+high does group: The cells were pre-treated with $20 \mu \mathrm{M}$ $\mathrm{Rb} 1$ for $24 \mathrm{~h}$, and then the cells were cultured in normal extracellular culture medium for $3 \mathrm{~h}$. (4) $\mathrm{Mg}^{2+}$ free+low does group: The cells were pre-treated with $5 \mu \mathrm{M} \mathrm{Rb} 1$ for $24 \mathrm{~h}$, and then the cells were cultured in $\mathrm{Mg}^{2+}$ free extracellular culture medium for $3 \mathrm{~h}$. (5) $\mathrm{Mg}^{2+}$ free+mid does group: The cells were pre-treated with $10 \mu \mathrm{M} \mathrm{Rb} 1$ for $24 \mathrm{~h}$, and then the cells were cultured in $\mathrm{Mg}^{2+}$ free extracellular culture medium for $3 \mathrm{~h}$. (6) $\mathrm{Mg}^{2+}$ free+high does group: The cells were pre-treated with $20 \mu \mathrm{M} \mathrm{Rb} 1$ for $24 \mathrm{~h}$, and then the cells were cultured in $\mathrm{Mg}^{2+}$ free extracellular culture medium for $3 \mathrm{~h}$.

\section{Nrf2 siRNA transfection}

Nrf2-siRNA and control siRNA were purchased from GenePharma (Shanghai, China). Hippocampal neurones were plated at a density of $1 \times 10^{5}$ cells/well in 12 -well plates $24 \mathrm{~h}$ prior to transfection. Then they were transfected with either Nrf2 siRNA (50 nM) or control siRNA (50 nM) with Lipofectamine RNAi Max (Invitrogen, USA; Cat: 13778075) for $24 \mathrm{~h}$.

\section{Cell viability assays}

The cell viability was determined by using the MTT assay (Sigma-Aldrich, USA) according to manufacturer's instructions. In brief, the cells $\left(5 \times 10^{5}\right.$ cells/well $)$ were plated on 96 -well plates. Then 100 $\mu \mathrm{l}$ of MTT $(5 \mathrm{mg} / \mathrm{mL})$ was added to each well and incubated for $4 \mathrm{~h}$ at 370. After that, the medium was removed and the $100 \mu \mathrm{l}$ DMSO solution was added in each well for another $30 \mathrm{~min}$ at 370. The OD value of each well was detected using a SpectraMax M5 Microplate Reader (Molecular Deviced, USA) at $570 \mathrm{~nm}$. 
Annexin V-FITC/ PI staining

Cell apoptosis/necrosis was evaluated using Annexin V-FITC/ PI staining as previously described [30, 31]. Briefly, cells were harvested and resuspended in binding buffer at a density of $1 \times 10^{6} \mathrm{cells} / \mathrm{mL}$. Then 100 $\mu \mathrm{l}$ of the sample solution was mixed with $5 \mu \mathrm{l}$ of Annexin V-FITC (Pharmingen) and $10 \mu \mathrm{l}$ of PI (Pharmingen) for $15 \mathrm{~min}$ in the dark at room temperature. The samples were analyzed by FACS (Becton Dickinson) using Cell Quest Research Software (Becton Dickinson). Annexin $\mathrm{V}^{-} / \mathrm{PI}^{-}$cells are viable, annexin $\mathrm{V}^{+} / \mathrm{PI}^{-}$cells are in early apoptosis and annexin $\mathrm{V}^{+} / \mathrm{PI}^{+}$cells are necrotic or in late apoptosis $[32,33]$.

\section{Western blot analysis}

Hippocampal tissues and cells were homogenized in lysis buffer (Roche, USA). The protein contents were determined by BCA assay (BioRad, USA). $40 \mu \mathrm{g}$ of proteins were electrophoreitically separated by SDS-PAGE and transferred on to PVDF membrane. After blocked in 3\% BSA/TBST at room temperature for $60 \mathrm{~min}$, the membranes were incubated with specific primary antibodies at $4^{\circ} \mathrm{C}$ overnight. The primary antibodies included Nrf2 (1: 800; Santa Cruz Biotechnology), HO-1 (1: 800; Santa Cruz Biotechnology), iNOS (1: 1000; Cell Signaling), Bcl-2 (1: 200, Santa Cruz Biotechnology) and LC3 (1: 500; Cell Signaling). GAPDH (1: 2000; Santa Cruz Biotechnology) was used to demonstrate equal protein loading. After washing with TBST, the membranes were incubated with horseradish-peroxidase conjugated secondary antibody (1: 2000). Finally, the immunoreactive bands were detected using an enhanced chemiluminescence method (Pierce).

\section{Statistical analysis}

The results are expressed as mean \pm SD. The results were analyzed statistically by using SPSS statistical package (version 16.0). The difference between two groups was compared using Student's $t$-test. Multigroup comparisons were carried out using two-way ANOVA followed by Bonferroni post hoc tests. Values of $P<$ 0.05 were considered statistically significant. All experiments were repeated at least for 3 times.

\section{Results}

\section{Rb1 antagonized PTZ-induced seizure activity}

Firstly, we evaluated Rb1 treatment on seizure activity induced by PTZ, which is used to elicit convulsive seizure activity. Seizure activity was graded based on the Racine scale, and presented as total seizure duration at stage $\mathrm{V}$ and the latency to stage $\mathrm{V}$. We found that the Rb1 (15, 30 and $60 \mathrm{mg} / \mathrm{kg}$ ) treated group significantly shortened PTZ-induced duration of seizures (Fig. 1A). In addition, the PTZ model of rat treated with the mid dose of Rb1 (30 mg/ $\mathrm{kg}$ ) and high dose of Rb1 (60 mg/kg) displayed dramatically prolonged latency compared with the PTZ+0.9\% NaCl group (Fig. 1B). However, there were no significant differences in latency and seizure duration between $\mathrm{PTZ}$ and $\mathrm{PTZ}+0.9 \% \mathrm{NaCl}$ group. Thus, treatment with $\mathrm{Rb} 1$ dose-dependently reduced PTZ-induced seizure severity.

\section{Rb1 attenuated PTZ-induced oxidative stress}

The levels of the GSH in the PTZ-treated group were significantly reduced by $66.67 \%$ of the normal group, which was significantly increased by the Rb1 treatment (Fig. 1C). By contrast, MDA levels in the hippocampus tissues were markedly elevated by about 2 folds in the PTZ-treated group when compared with that of the normal group. The increased MDA level was notably attenuated in the group co-treated with Rb1 (Fig. 1D). However, there were no significant differences in GSH and MDA levels between PTZ and PTZ + 0.9\% $\mathrm{NaCl}$ group.

\section{Rb1 improved PTZ-induced defects in cognitive function}

Next, we investigated whether Rb1 affected performance in a Morris water maze test. Swimming speed and distance and escape latencies in the MWM were used to evaluate the cognitive function. We found that there were no significant differences in average swim speeds between the treatment groups (Fig. 2A). As shown in Fig. 2B, PTZ-treated animals had significantly longer escape latencies compared to those in normal group. However, the 


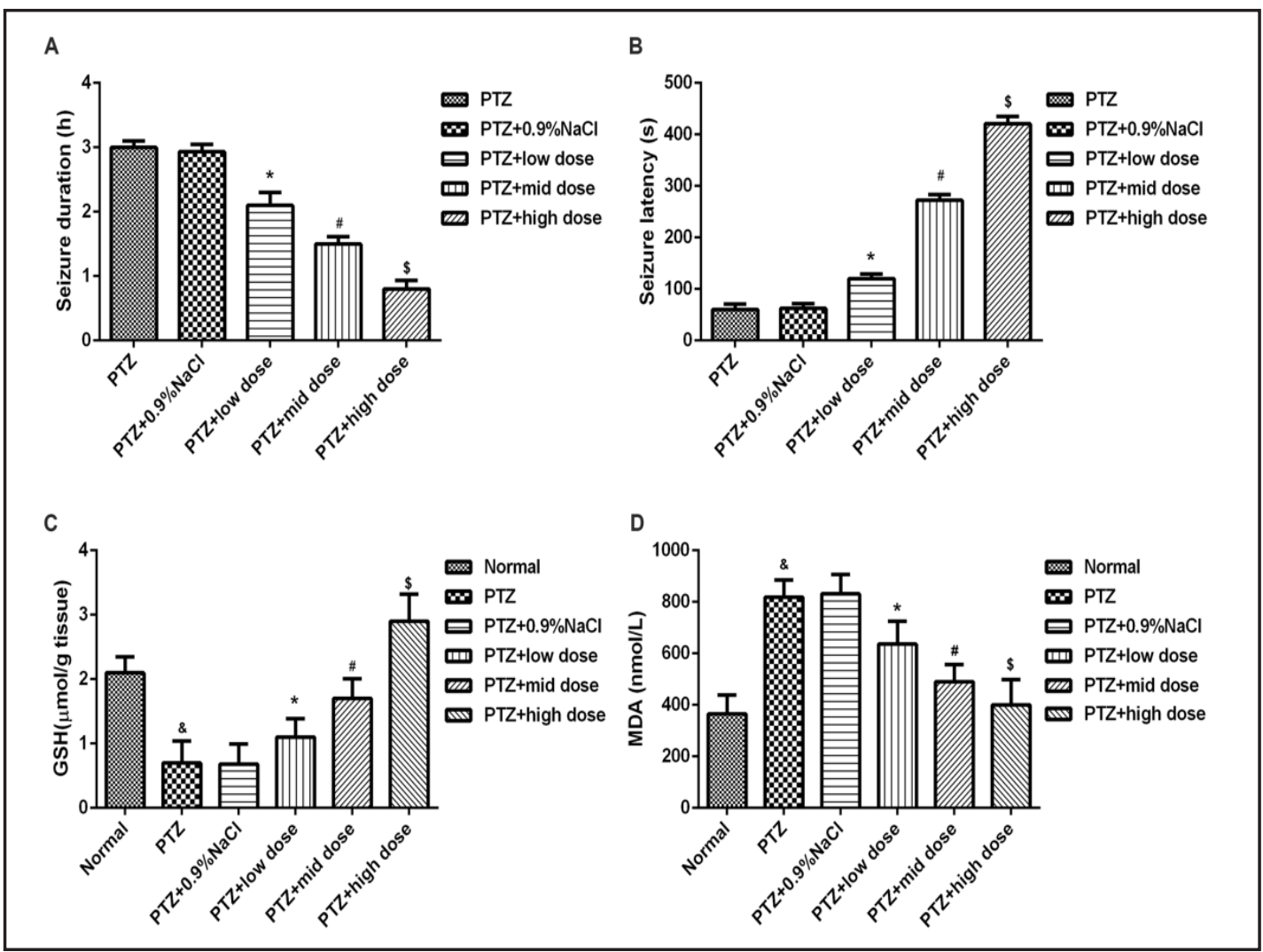

Fig. 1. Effects of $\mathrm{Rb} 1$ on seizure duration and latency and oxidative stress damage in the PTZ-treated rats. (A) Behavioral seizures induced by PTZ were presented as average seizure duration at grade $V(n=6)$. (B) The latency to grade $V(n=6)$. (C) GSH levels (n=6). (D) MDA levels $(n=6) .{ }^{*} \mathrm{P}<0.05$ and ${ }^{* *} \mathrm{P}<0.01$ vs. PTZ group, ${ }^{* * *} \mathrm{P}<0.001$ vs. normal group, ${ }^{\#} \mathrm{P}<0.05$ and ${ }^{\# \#} \mathrm{P}<0.01$ vs. $\mathrm{PTZ}+0.9 \% \mathrm{NaCl}$ group.

increased escape latencies were reduced by Rb1 in a dose-dependent manner. Additionally, the increased swimming distances induced by PTZ were significantly blocked by Rb1 (Fig. 2C). These findings indicated that $\mathrm{Rb} 1$ dose-dependently reduced PTZ-induced impaired cognitive function of the rats.

\section{Rb1 alleviated neuronal damage in rats exposed to PTZ treatment}

As shown in Fig. 3A, H\&E staining revealed that after PTZ treatment, the arrangement of neurons was irregular, and the neurons exhibited pyknosis, which is characterized by a darkly stained nucleus and cytoplasm. However, the number of abnormal neurons was significantly decreased in the groups treated with Rb1. Nissl staining indicated neuronal injuries. After PTZ treatment, nuclear pyknosis, cell loss and karyorrhexis were observed. Exposure to 15, 30 and $60 \mathrm{mg} / \mathrm{kg} \mathrm{Rb1}$ alleviated the neuronal injury caused by PTZ (Fig. 3A). Compared with the $\mathrm{PTZ}+0.9 \% \mathrm{NaCl}$ group, the density of normal neurons in the Rb1 treatment groups were clearly increased. However, there was no significant difference between the PTZ and PTZ + $0.9 \% \mathrm{NaCl}$ group.

$R b 1$ reduced neuronal apoptosis and oxidative damage and restored autophagy through the Nrf2/HO-1 pathway in PTZ-treated rats

As shown in Fig. 3B, PTZ treatment decreased Bcl-2 protein expression, whereas iNOS and LC3 expression increased than that of the normal group. Interestingly, addition of Rb1 suppressed the level of iNOS and LC3 expression, increased the levels of Bcl-2 expression in PTZ-treated rats. 


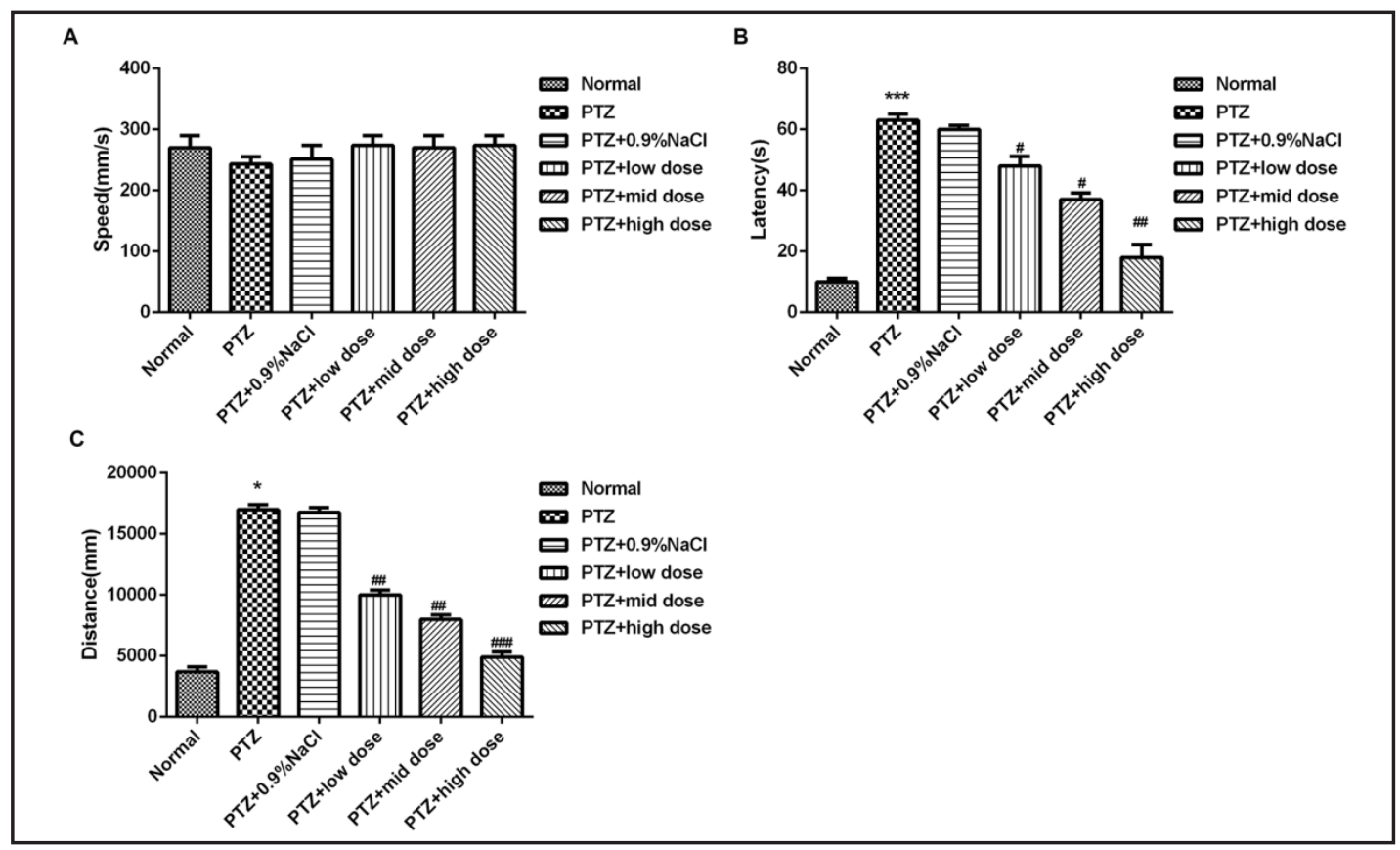

Fig. 2. Effects of Rb1 on cognitive function in the PTZ-treated rats. (A) Swimming speed ( $n=6)$. (B) Latency to locate the platform (escape latency; $\mathrm{n}=6$ ). (C) Swimming distance $(\mathrm{n}=6) .{ }^{*} \mathrm{P}<0.05$ and ${ }^{* * *} \mathrm{P}<0.001$ vs. normal group, ${ }^{\mathrm{P}}<0.05,{ }^{\# \#} \mathrm{P}<0.01$ and ${ }^{\# \# \#} \mathrm{P}<0.01$ vs. $\mathrm{PTZ}+0.9 \% \mathrm{NaCl}$ group.

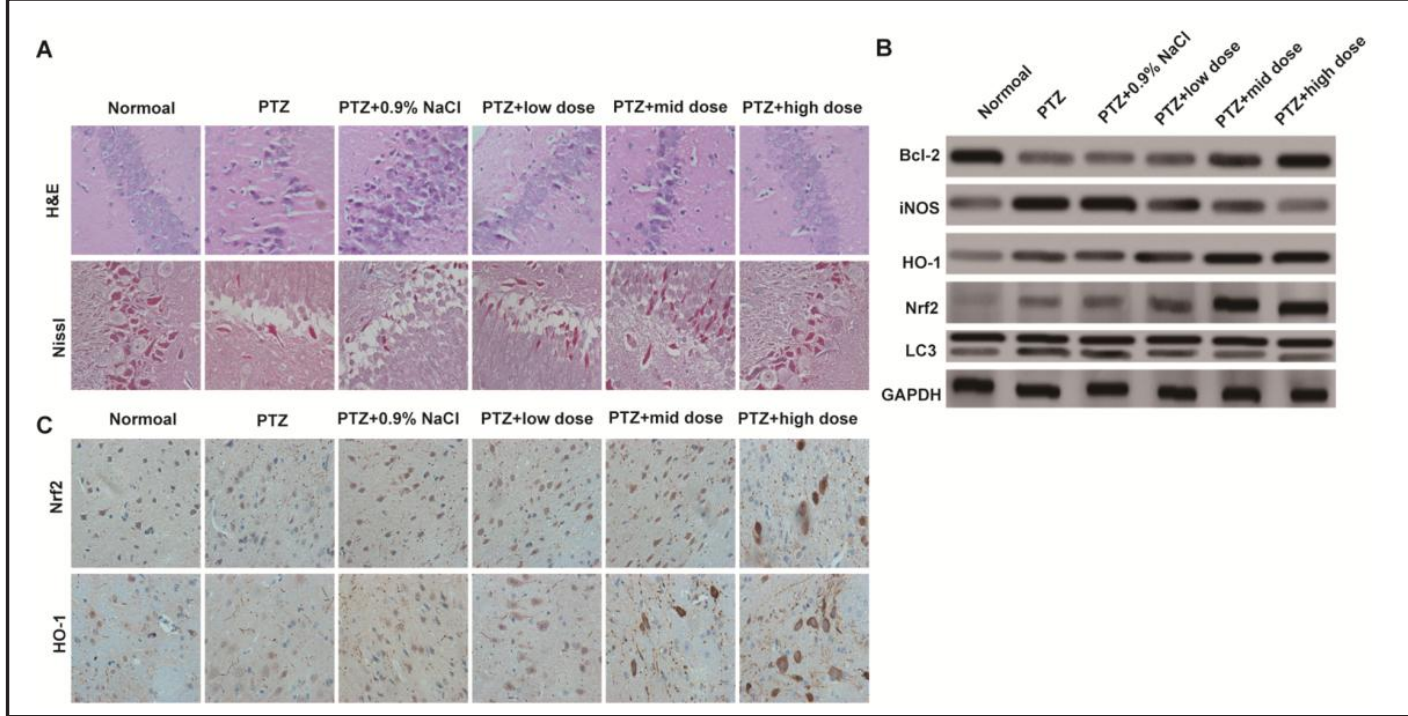

Fig. 3. Effects of $\mathrm{Rb} 1$ on neuronal damage, apoptosis, oxidative damage, autophagy and protein expression levels of Nrf2 and HO-1 in the PTZ-treated rats. (A) Representative H\&E staining and Nissl staining in the CA1 region of the hippocampus (200 $\times$ magnification; $n=3)$. (B) Representative western blot images to detect the expressions of Bcl-2, iNOS, Nrf2, HO-1 and LC3. The experiment repeated three times. (C) The expression of Nrf2 and HO-1 in the groups by immunohistochemmistry staining (200 × magnification; $\mathrm{n}=3)$.

Subsequently, we hypothesized that Nrf2/HO-1 signaling pathways were involved in the neuroprotective effect induced by $\mathrm{Rb} 1$. We examined the protein levels of HO-1 and Nrf2 treated with different doses of Rb1 in PTZ-treated rats by western blot assay. Our data revealed that PTZ-treated rat showed an increase of Nrf2 and HO-1 expression levels. And the increased Nrf2 and HO-1 expression levels induced by PTZ were enhanced by Rb1 (Fig. 3B). In addition, we analyzed the expression patterns of Nrf2 and HO-1 in hippocampus KARGER 


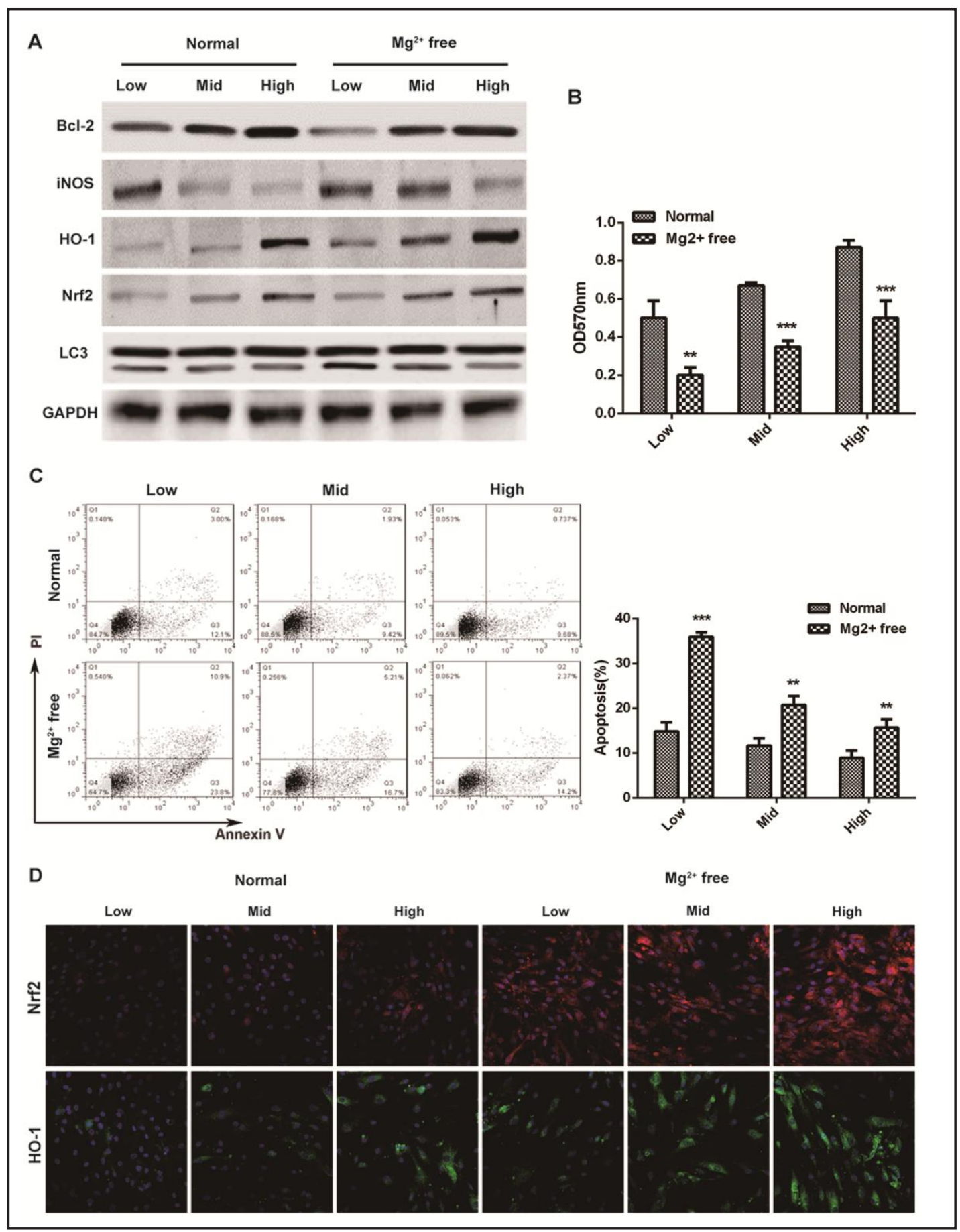

Fig. 4. Effects of $\mathrm{Rb} 1$ on neuronal apoptosis, oxidative damage, autophagy and protein expression levels of Nrf2 and HO-1 in hippocampal neurons under $\mathrm{Mg}^{2+}$ free condition. (A) Representative western blot images to detect the expressions of Bcl-2, iNOS, Nrf2, HO-1 and LC3. The experiment repeated three times. (B) Cell viability was assessed by MTT assay $(n=3)$. (C) Cells were double stained with annexin V-FITC and PI and analyzed by flow cytometry. And percentage of apoptotic cells was shown by histogram (n=3). (D) The expression of Nrf2 (red) and HO-1 (green) in the groups by immunofluorescence staining $(200 \times$ magnification; $n=3$ ). ${ }^{* *} \mathrm{P}<0.01$ and ${ }^{* * *} \mathrm{P}<0.001$ vs. their corresponding normal group treated with low dose, mid dose and high dose $\mathrm{Rb} 1$. 


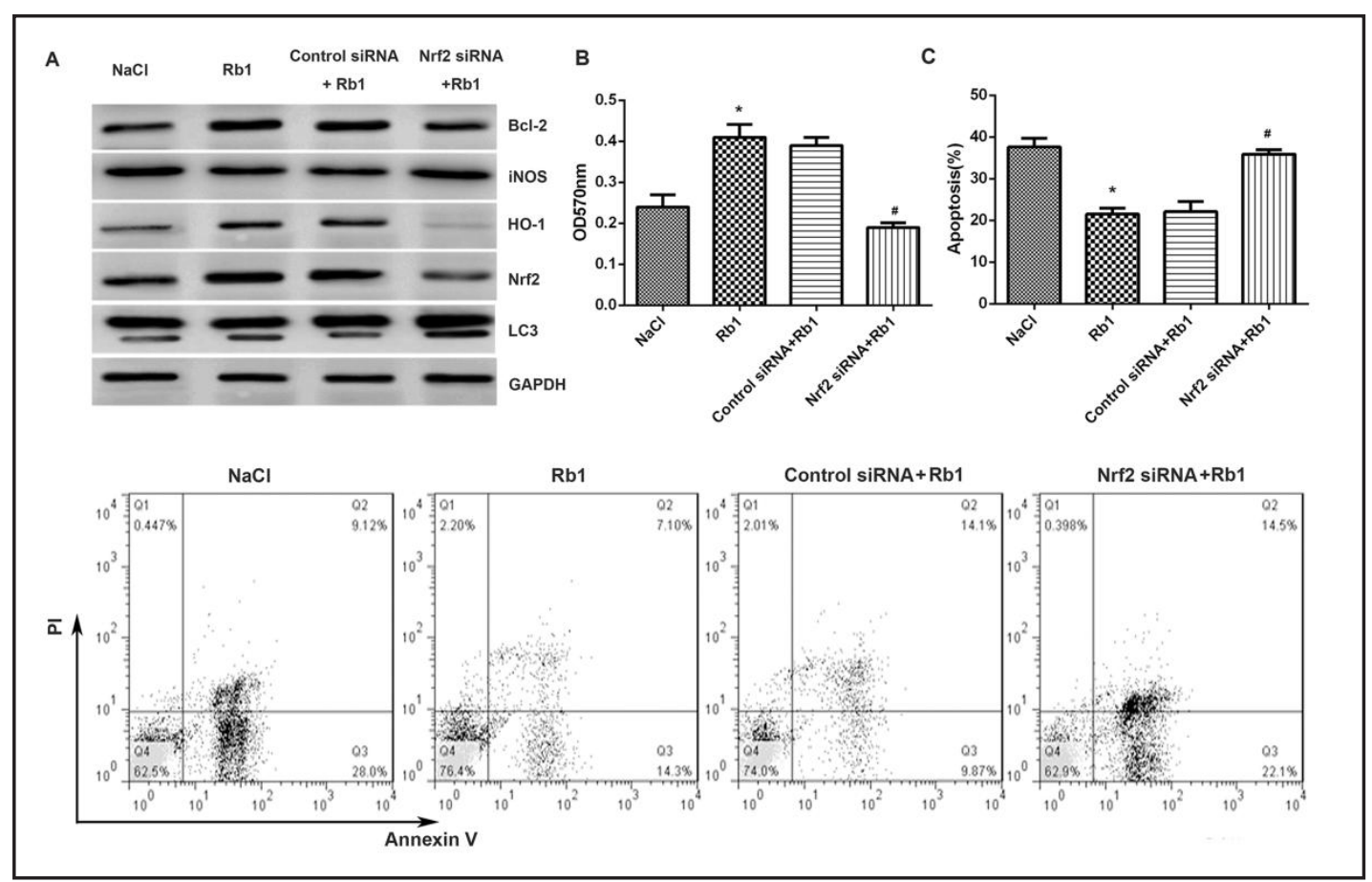

Fig. 5. Knockdown of Nrf2 blocked the effects of Rb1 on neuronal apoptosis, oxidative damage and autophagy in hippocampal neurons under $\mathrm{Mg}^{2+}$ free condition. The cells were transfected with either Nrf2 siRNA or control siRNA. The transfected cells were pre-treated with $20 \mu \mathrm{M} \mathrm{Rb} 1$ for $24 \mathrm{~h}$, and then the cells were cultured in $\mathrm{Mg}^{2+}$ free extracellular culture medium for $3 \mathrm{~h}$. The untransfected cells under $0.9 \% \mathrm{NaCl}$ or $20 \mu \mathrm{M} \mathrm{Rb} 1$ for $24 \mathrm{~h}$ and then cultured in normal extracellular culture medium for $3 \mathrm{~h}$ were used as control. (A) Representative western blot images to detect the expressions of Bcl-2, iNOS, Nrf2, HO-1 and LC3. The experiment repeated three times. (B) Cell viability was assessed by MTT assay (n=3). (C) Cells were double stained with annexin V-FITC and PI and analyzed by flow cytometry. And percentage of apoptotic cells was shown by histogram $(\mathrm{n}=3)$. ${ }^{*} \mathrm{P}<0.05$ vs. $\mathrm{NaCl}$ group, ${ }^{*} \mathrm{P}<0.05$ vs. $\mathrm{Rb} 1+$ control siRNA group.

tissues by immunohistochemical staining (Fig. 3C). The immunohistochemical analysis showed the positive staining of Nrf2 and HO-1 proteins was increased in PTZ group (Fig. 3C). After Rb1 treatment, the Nrf2 and HO-1 protein levels were significantly higher than that in the PTZ+0.9\% NaCl group.

$R b 1$ reduced neuronal apoptosis and oxidative damage and restored autophagy through the Nrf2/HO-1 pathway in epileptic hippocampal neurons

To evaluate the neuroprotective effects of $\mathrm{Rb} 1$, western blot was performed on epileptic neurons that were exposed to different concentrations of Rb1. As shown in Fig. 4A, Rb1 led to a dose-dependent increase in the protein expression of Bcl-2 and a decrease in the protein expression of iNOS and LC3 in both the normal neurons and the $\mathrm{Mg}^{2+}$-free treated neurons. Moreover, the expression levels of iNOS were higher in $\mathrm{Mg}^{2+}$-free treated group compared with that in corresponding normal group.

Subsequently, the results from the MTT assay (Fig. 4B) showed that Rb1 induced an increase in cell viability in a dose-dependent manner in both the normal neurons and the $\mathrm{Mg}^{2+}$-free treated neurons. However, the cell viability was significantly lower in $\mathrm{Mg}^{2+}-$ free treated group compared with that in corresponding normal group. Furthermore, the anti-apoptotic effects of Rb1 were further investigated using an Annexin V-FITC/PI assay. Treatment with $\mathrm{Rb} 1$ dose-dependently attenuated cell apoptosis (Fig. 4C).

Next, we studied whether Nrf2/HO-1 signaling pathways were involved in the neuroprotective effect of $\mathrm{Rb} 1$. Western blot revealed that $\mathrm{Rb} 1$ treatment dramatically increased the Nrf2 and HO-1 expression levels in both the normal neurons and the $\mathrm{Mg}^{2+}$-free 
treated neurons (Fig. 4A). Besides, we detected the expression patterns of Nrf2 and HO-1 by immunofluorescence staining (Fig. 4D). Moreover, the fluorescence intensity of the Nrf2 and HO-1 was significantly increased after Rb1 treatment.

Knockdown of Nrf2 blocked the neuroprotective effects of Rb1 in epileptic hippocampal neurons

To further confirm the roles of Nrf2/HO-1 pathway in the neuroprotective effects of $\mathrm{Rb} 1$, epileptic hippocampal neurons were transfected with Nrf2 siRNA to knock down Nrf2 expression. As shown in Fig. 5A, Rb1 significantly increased the Nrf2 and HO-1 protein expression, where the effect was ameliorated by transfection with Nrf2 siRNA. Moreover, knockdown of Nrf2 repressed Rb1-induced upregulation of $\mathrm{Bcl}-2$ and reduced the downregulation of the protein expression of iNOS and LC3 (Fig. 5A).

Next, MTT assay showed that Rb1 induced an increase in cell viability in the cells transfected with the control siRNA, which was blocked by Nrf2 siRNA (Fig. 5B). In addition, inhibition of $\mathrm{Nrf} 2$ attenuated $\mathrm{Rb} 1$ induced decrease of the percentage of apoptotic cells (Fig. 5C).

\section{Discussion}

PTZ, an epilepsy inducing agent, is a $\gamma$-aminobutyric acid (GABA) receptor antagonist, which has high biological membrane permeability and consequent rapid bioavailability and distribution to all organs including the brain [34, 35]. Moreover, PTZ-induced chemical kindling in rats is an accepted model for identifying antiepileptic drugs [36]. Bolkvadze et al. reported that in the PTZ test, the latency to the first seizure in mice with hippocampal damage was reduced compared with the mice without damage [37]. Hussein et al. demonstrated that PTZ kindling was associated with behavioral changes, which were in the form high stage of Racine score, long seizure duration and short latency for the first jerk [38]. Our results found that $\mathrm{Rb} 1$ dose-dependently reduced PTZ-induced seizure duration and prolonged seizure latency, suggesting Rb1 can suppress the severity of PTZ-induced seizures.

Seizure is also associated with cognitive impairment [39], which can be demonstrated by the animals' poor performance in the Morris water maze test. Our findings were consistent with previous studies, indicating that cognitive functions of animals significantly declined after seizure, as indicated by the significant increase in escape latency obtained in MWM [39, 40]. Rb1-treated PTZ kindled rat improved performance in identification of hidden platform, as demonstrated by shorter escape latency and shorter swimming distance. And previous studies have revealed that Rb1 improves spatial learning in rat, as evidenced by spending less time in finding the hidden platform with repeated training [41]. These observations suggest that Rb1 improves cognition of PTZ kindled rat.

Oxidative stress has been reported to contribute the development and progression of epilepsy [42]. The increased free radicals lead to membrane lipid peroxidation and reduced glutathione concentrations in the epileptic focus [43]. In the present study, PTZ-induced seizures caused a marked decrease in GSH levels and a significant increase in MDA levels compared to the normal group. In agreement with our results, the PTZ-induced oxidative stress has been reported in previous studies [44, 45]. However, these changes were ameliorated by $\mathrm{Rb} 1$. The antioxidant effects of $\mathrm{Rb} 1$ have been reported in previous studies $[46,47]$. Thus, our results implied that $\mathrm{Rb} 1$ exerts a neuroprotective effect against PTZinduced injuries by alleviating oxidative stress.

Histopathological studies of the hippocampus tissues indicated that epilepsy induced by PTZ caused an obvious decrease in the number of the normal neurons, as evidenced by a decrease in its Nissl granules content compared with normal group, which was in agreement with previous studies [48]. However, Rb1 administration in the present study displayed a decrease in hippocampal damage through a significant increase in the Nissl granules content of the normal neurons, indicating that Rb1 protects against PTZ-induced hippocampal neuron damage.

\section{KARGER}


To further detect the possible mechanism involved in the neuroprotective effects of $\mathrm{Rb} 1$, we analyzed the expression of Bcl-2, iNOS and LC3. Apoptosis is modulated by multiple signaling pathways in which Bcl-2 protein families play critical roles [49]. In addition, Bcl2 is an anti-apoptotic protein that can reduce apoptotic cell death [50]. PTZ treatment caused a significant decrease in the expression of Bcl-2 proteins in the brain tissues of mice [51]. Similar results were also found in our studies. Moreover, iNOS, which produce amounts of nitric oxide (NO), can create an oxidative stress [52]. Our findings indicate that $\mathrm{Rb} 1$ treatment dramatically decreased iNOS levels in PTZ induced seizures, which indicates the antioxidant effect of Rb1. Furthermore, LC3, a crucial protein for autophagy, and LC3 immunoblot analysis is now widely used to monitor autophagy, because the ratio of LC3II/I is associated with the number of autophagosomes [53]. Our data confirmed the results of previous studies that a significant increase in the expression of LC3 in the hippocampal region of fully PTZ-kindled rat [54]. However, Rb1 restored the elevation of LC3, indicating that $\mathrm{Rb} 1$ treatment could restore autophagy after PTZ treatment.

In vitro, we found that Rb1 reduced iNOS and LC3 expression and increased the Bcl2 expression in epileptic hippocampal neurons, demonstrating that $\mathrm{Rb} 1$ could alleviate oxidative damage and autophagy and neuron apoptosis under $\mathrm{Mg}^{2+}$ free condition. The present results confirmed the mechanisms of $\mathrm{Rb} 1$ protective effects mentioned in vivo.

In order to confirm that the neuroprotective effects of Rb1 were dependent on Nrf2/ ARE signaling, we detected the effects of Rb1 on activation of the Nrf2 and HO-1 genes in vivo and in vitro. Previous studies have demonstrated that the expression of Nrf2 and HO-1 at protein and mRNA levels markedly increased in hippocampus of amygdala kindling rats $[55,56]$. Moreover, activation of Nrf2-ARE signal pathway in hippocampus reduced the progression of amygdale kindling, and alleviated the cognitive impairment and oxidative stress induced by epileptic seizure [21,57]. Thus, Nrf2-ARE pathway may bring great benefit to the brain damage after seizure. Our results showed that after PTZ treatment in vivo and $\mathrm{Mg}^{2+}$ free cultivation in vitro, the expressions of Nrf2 and HO-1 were significantly increased. In addition, $\mathrm{Rb} 1$ promoted the elevation of Nrf2 and HO-1 in a dose-dependent manner. However, knockdown of Nrf2 blocked the protective effects of Rb in epileptic hippocampal neurons. Therefore, $\mathrm{Rb} 1$ attenuates brain damage induced by epileptic seizure by activating the Nrf2/ARE pathway.

In summary, our data showed that Rb1 could ameliorate seizure severity and the cognitive impairment. In addition, the present study suggested that $\mathrm{Rb} 1$ also reduces the oxidative stress and neuron apoptosis and restores autophagy induced by epileptic seizure through the Nrf2/ARE pathway. Thus, Rb1 can represent a novel anti-epileptic drug for therapy in epilepsy.

\section{Disclosure Statement}

All the authors declared no conflicts of interest.

\section{References}

1 Dua T, Boer HMD, Prilipko LL, Saxena S: Epilepsy Care in the World: Results of an ILAE/IBE/WHO Global Campaign Against Epilepsy Survey. Epilepsia 2006;47:1225-1231.

2 Moshé SL, Perucca E, Ryvlin P, Tomson T: Epilepsy: new advances. Lancet 2015;385:884-898.

-3 Berkovic SF, Mulley JC, Scheffer IE, Petrou S: Human epilepsies: interaction of genetic and acquired factors. Trends Neurosci 2006;29:391-397.

4 John McMahon XH, Jun Yang, Masaaki Komatsu, Zhenyu Yue, Jiang Qian, Xinjun Zhu, Yunfei Huang: Impaired autophagy in neurons following disinhibtion of mTOR and its contribution to epileptogenesis. J Neurosci 2012;32:15704. 


\section{Cellular Physiology Cell Physiol Biochem 2018;45:212-225 \\ \begin{tabular}{l|l|l|} 
DOI: 10.1159/000486768 & $\begin{array}{l}\text { O } 2018 \text { The Author(s). Published by S. Karger AG, Basel } \\
\text { www.karger.com/cpb }\end{array}$ \\
\hline
\end{tabular}}

Shi et al.: Ginsenoside Rb1, a Novel Anti-Epileptic Drug

5 Puttachary S, Sharma S, Stark S, Thippeswamy T: Seizure-Induced Oxidative Stress in Temporal Lobe Epilepsy. BioMed Res Int 2014;2015:796-802.

6 Xu D, Miller SD, Koh S: Immune mechanisms in epileptogenesis. Front Cell Neurosci 2013;7:195.

7 Kaminski RM, Rogawski MA, Klitgaard H: The Potential of Antiseizure Drugs and Agents that Act on Novel Molecular Targets as Antiepileptogenic Treatments. Neurotherapeutics 2014;11:385-400.

-8 Wang L, Lu AP, Yu ZL, Wong RN, Bian ZX, Kwok HH, Yue PY, Zhou LM, Chen H, Xu M: The melanogenesisinhibitory effect and the percutaneous formulation of ginsenoside Rb1. AAPS PharmSciTech 2014;15:12521262.

-9 Liu D, Zhang H, Gu W, Liu Y, Zhang M: Neuroprotective effects of ginsenoside Rb1 on high glucose-induced neurotoxicity in primary cultured rat hippocampal neurons. PLoS One 2013;8:e79399-e79399.

10 Zhu J, Jiang Y, Wu L, Lu T, Xu G, Liu X: Suppression of local inflammation contributes to the neuroprotective effect of ginsenoside Rb1 in rats with cerebral ischemia. Neuroscience 2012;202:342-351.

11 Gao XQ, Yang CX, Chen GJ, Wang GY, Bo C, Tan SK, Liu J, Yuan QL: Ginsenoside Rb1 regulates the expressions of brain-derived neurotrophic factor and caspase-3 and induces neurogenesis in rats with experimental cerebral ischemia. J Ethnopharmacol 2010;132:393.

12 Liang J, Yao J, Wang G, Wang Y, Wang B, Ge P: Ischemic Postconditioning Protects Neuronal Death Caused by Cerebral Ischemia and Reperfusion via Attenuating Protein Aggregation. Int J Med Sci 2012;9:923-932.

13 Liu L, Hoang-Gia T, Wu H, Lee MR, Gu L, Wang C, Yun BS, Wang Q, Ye S, Sung CK: Ginsenoside Rb1 improves spatial learning and memory by regulation of cell genesis in the hippocampal subregions of rats. Brain Res 2011;1382:147-154.

14 Xing HY, Cai YQ Wang XF, Wang LL, Pan L, Wang GY, Chen JH: The Cytoprotective Effect of Hyperoside against Oxidative Stress Is Mediated by the Nrf2-ARE Signaling Pathway through GSK-3 $\beta$ Inactivation. PLoS One 2015;10:e0145183.

15 Li X, Han D, Tian Z, Gao B, Fan M, Li C, Wang Y, Ma S, Cao F: Activation of Cannabinoid Receptor Type II by AM1241 Ameliorates Myocardial Fibrosis via Nrf2-Mediated Inhibition of TGF- $\beta 1 /$ Smad3 Pathway in Myocardial Infarction Mice. Cell Physiol Biochem 2016;39:1521-1536.

16 Zhang H, Liu H, Davies KJ, Sioutas C, Finch CE, Morgan TE, Forman HJ: Nrf2-regulated phase II enzymes are induced by chronic ambient nanoparticle exposure in young mice with age-related impairments. Free Radic Biol Med 2012;52:2038-2046.

17 Itoh K, Tong KI, Yamamoto M: Molecular mechanism activating Nrf2-Keap1 pathway in regulation of adaptive response to electrophiles. Free Radic Biol Med 2004;36:1208-1213.

-18 Colín-González AL, Orozco-Ibarra M, Chánez-Cárdenas ME, Rangel-López E, Santamaría A, PedrazaChaverri J, Barrera-Oviedo D, Maldonado PD: Heme oxygenase-1 (HO-1) upregulation delays morphological and oxidative damage induced in an excitotoxic/pro-oxidant model in the rat striatum. Neuroscience 2013;231:91-101.

19 Zhong Z, Tang Y: Upregulation of Periostin Prevents High Glucose-Induced Mitochondrial Apoptosis in Human Umbilical Vein Endothelial Cells via Activation of Nrf2/HO-1 Signaling. Cell Physiol Biochem 2016;39:71-80.

-20 Calkins MJ, Johnson DA, Townsend JA, Vargas MR, Dowell JA, Williamson TP, Kraft AD, Lee JM, Li J, Johnson JA: The Nrf2/ARE pathway as a potential therapeutic target in neurodegenerative disease. Antioxid Redox Signal 2009;11:497-508.

-21 Wang W, Wu Y, Zhang G, Fang H, Wang H, Zang H, Xie T: Activation of Nrf2-ARE signal pathway protects the brain from damage induced by epileptic seizure. Brain Res 2013;1544:54-61.

22 Liu W, Wang B, Wang T, Liu X, He X, Liu Y, Li Z, Zeng H: Ursodeoxycholic Acid Attenuates Acute Aortic Dissection Formation in Angiotensin II-Infused Apolipoprotein E-Deficient Mice Associated with Reduced ROS and Increased Nrf2 Levels. Cell Physiol Biochem 2016;38:1391-1405.

23 Racine RJ: Modification of seizure activity by electrical stimulation: II. Motor seizure. Electroencephalogr Clin Neurophysiol 1972;32:269-279.

24 Chen Z, Tao ZZ, Chen SM, Chen C, Li F, Xiao BK: Indole-3-carbinol inhibits nasopharyngeal carcinoma growth through cell cycle arrest in vivo and in vitro. PLoS One 2012;8:e82288.

25 Yu SS, Zhao J, Zheng WP, Zhao Y: Neuroprotective effect of 4-hydroxybenzyl alcohol against transient focal cerebral ischemia via anti-apoptosis in rats. Brain Res 2009;1308:167-175.

-26 Wen M, Yan Y, Yan N, Chen XS, Liu SY, Feng ZH: Upregulation of RBFOX1 in the malformed cortex of patients with intractable epilepsy and in cultured rat neurons. Int J Mol Med 2015;35:597-606. 


\section{Cellular Physiology Cell Physiol Biochem 2018;45:212-225 \\ \begin{tabular}{l|l|l|} 
DOI: 10.1159/000486768 & $\begin{array}{l}\text { O } 2018 \text { The Author(s). Published by S. Karger AG, Basel } \\
\text { www.karger.com/cpb }\end{array}$ \\
\hline
\end{tabular}}

Shi et al.: Ginsenoside Rb1, a Novel Anti-Epileptic Drug

-27 Irving AJ, Collingridge GL: A characterization of muscarinic receptor-mediated intracellular Ca2+ mobilization in cultured rat hippocampal neurones. J Physiol (Lond) 1998;511:747-759.

-28 Raol YH, Brookskayal AR: Experimental models of seizures and epilepsies. Prog Mol Biol Transl Sci 2012;105:57-82.

29 Sombati S, Delorenzo RJ: Recurrent spontaneous seizure activity in hippocampal neuronal networks in culture. J Neurophysiol 1995;73:1706-1711.

30 Chen S, Cheng AC, Wang MS, Peng X: Detection of apoptosis induced by new type gosling viral enteritis virus in vitro through fluorescein annexin V-FITC/PI double labeling. World J Gastroenterol 2008;14:21742178.

31 Xia C, Bai X, Hou X, Gou X, Wang Y, Zeng H, Huang M, Jin J: Cryptotanshinone Reverses Cisplatin Resistance of Human Lung Carcinoma A549 Cells through Down-Regulating Nrf2 Pathway. Cell Physiol Biochem 2015;37:816-824.

-32 Freire ACG, Assis CFD, Frick AO, Melo PCDS, Haun M, Aoyama H, Durán N, Sauer MM, Kallás ÉG, Ferreira CVS: Influence of protein phosphatase inhibitors on HL60 cells death induction by dehydrocrotonin. Leuk Res 2003;27:823-829.

33 Ming X, Li XX, Lei W, Mi W, Yang Z, Li PL: Contribution of Nrf2 to Atherogenic Phenotype Switching of Coronary Arterial Smooth Muscle Cells Lacking CD38 Gene. Cell Physiol Biochem 2015;37:432-444.

-34 Zienowicz M, Wisłowska A, Lehner M, Taracha E, Skórzewska A, Maciejak P, Płaźnik A: The effect of fluoxetine in a model of chemically induced seizures--behavioral and immunocytochemical study. Neurosci Lett 2005;373:226-231.

35 Konishi Y, Matsu-Ura T, Mikoshiba K, Tamura T: Stimulation of gene expression of NeuroD-related factor in the mouse brain following pentylenetetrazol-induced seizures. Brain Res Mol Brain Res 2001;97:129-136.

-36 Fischer W, Kittner H: Influence of ethanol on the pentylenetetrazol-induced kindling in rats. J Neural Transm 1998;105:1129-1142.

-37 Bolkvadze T, Pitkänen A: Development of post-traumatic epilepsy after controlled cortical impact and lateral fluid-percussion-induced brain injury in the mouse. J Neurotrauma 2012;29:789-812.

-38 Hussein AM, Ghalwash M, Magdy K, Abulseoud OA: Beta Lactams Antibiotic Ceftriaxone Modulates Seizures, Oxidative Stress and Connexin 43 Expression in Hippocampus of Pentylenetetrazole Kindled Rats. J Epilepsy Res 2016;6:8-15.

-39 Cardoso A, Lukoyanova EA, Madeira MD, Lukoyanov NV: Seizure-induced structural and functional changes in the rat hippocampal formation: Comparison between brief seizures and status epilepticus. Behav Brain Res 2011;225:538-546.

40 Hao F, Jia LH, Li XW, Zhang YR, Liu XW: Garcinol Upregulates GABAA and GAD65 Expression, Modulates BDNF-TrkB Pathway to Reduce Seizures in Pentylenetetrazole (PTZ)-Induced Epilepsy. Med Sci Monit 2016;22:4415-4425.

41 Mook-Jung I, Hong HS, Boo JH, Kang HL, Yun SH, Mi YC, Joo I, Huh K, Min WJ: Ginsenoside Rb1 and Rg1 improve spatial learning and increase hippocampal synaptophysin level in mice. J Neurosci Res 2001;63:509-515.

42 Sudha K, Rao AV, Rao A: Oxidative stress and antioxidants in epilepsy. Clin Chim Acta 2001;303:19-24.

43 Jesberger JA, Richardson JS: Oxygen free radicals and brain dysfunction. Int J Neurosci 1991;57:1-17.

-44 Agarwal NB, Agarwal NK, Mediratta PK, Sharma KK: Effect of lamotrigine, oxcarbazepine and topiramate on cognitive functions and oxidative stress in PTZ-kindled mice. Seizure 2011;20:257-262.

45 Ilhan A, Aladag MA, Kocer A, Boluk A, Gurel A, Armutcu F: Erdosteine ameliorates PTZ-induced oxidative stress in mice seizure model. Brain Res Bull 2005;65:495-499.

46 Hwang YP, Jeong HG: Ginsenoside Rb1 protects against 6-hydroxydopamine-induced oxidative stress by increasing heme oxygenase-1 expression through an estrogen receptor-related PI3K/Akt/Nrf2-dependent pathway in human dopaminergic cells. Toxicol Appl Pharmacol 2009;242:18-28.

47 Zhuang CL, Mao XY, Liu S, Chen WZ, Huang DD, Zhang CJ, Chen BC, Shen X, Yu Z: Ginsenoside Rb1 improves postoperative fatigue syndrome by reducing skeletal muscle oxidative stress through activation of the PI3K/Akt/Nrf2 pathway in aged rats. Eur J Pharmacol 2014;740:480-487.

48 Haggag BS, Hasanin AH, Raafat MH, Abdel Kawy HS: Lamotrigine decreased hippocampal damage and improved vascular risk markers in a rat model of pentylenetetrazole induced kindling seizure. Korean J Physiol Pharmacol 2014;18:269-278. 


\section{Cellular Physiology Cell Physiol Biochem 2018;45:212-225

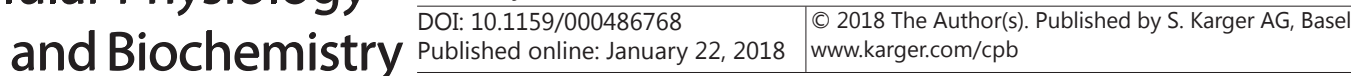

Shi et al.: Ginsenoside Rb1, a Novel Anti-Epileptic Drug

49 Siddiqui WA, Ahad A, Ahsan H: The mystery of BCL2 family: Bcl-2 proteins and apoptosis: an update. Arch Toxicol 2015;89:289-317.

50 Giordano G, Klintworth HM, Kavanagh TJ, Costa LG: Apoptosis induced by domoic acid in mouse cerebellar granule neurons involves activation of p38 and JNK MAP kinases. Neurochem Int 2008;52:1100-1105.

-51 Naseer MI, Ullah N, Ullah I, Koh PO, Lee HY, Park MS, Kim MO: Vitamin C protects against ethanol and PTZinduced apoptotic neurodegeneration in prenatal rat hippocampal neurons. Synapse 2011;65:562-571.

-52 Sun J, Druhan LJ, Zweier JL: Reactive Oxygen and Nitrogen Species Regulate Inducible Nitric Oxide Synthase Function Shifting the Balance of Nitric Oxide and Superoxide Production. Arch Biochem Biophys 2009;494:130-137.

53 Jiang H, White EJ, Conrad C, Gomezmanzano C, Fueyo J: Autophagy pathways in glioblastoma. Methods Enzymol 2009;453:273-286.

54 Zhu X, Shen K, Bai Y, Zhang A, Xia Z, Chao J, Yao H: NADPH oxidase activation is required for pentylenetetrazole kindling-induced hippocampal autophagy. Free Radic Biol Med 2016;94:230-242.

55 Wang W, Wang WP, Zhang GL, Wu YF, Xie T, Kan MC, Fang HB, Wang HC: Activation of Nrf2-ARE signal pathway in hippocampus of amygdala kindling rats. Neurosci Lett 2013;543:58-63.

56 Wang F, Pu C, Zhou P, Wang P, Liang D, Wang Q, Hu Y, Li B, Hao X: Cinnamaldehyde prevents endothelial dysfunction induced by high glucose by activating Nrf2. Cell Physiol Biochem 2015;36:315-324.

57 Shi L, Wu L, Chen Z, Yang J, Chen X, Yu F, Zheng F, Lin X: MiR-141 Activates Nrf2-Dependent Antioxidant Pathway via Down-Regulating the Expression of Keap1 Conferring the Resistance of Hepatocellular Carcinoma Cells to 5-Fluorouracil. Cell Physiol Biochem 2015;35:2333-2348. 